\title{
The vehicle driver safety prediction system
}

\section{ARTICLE INFO}

Received: 14 July 2021

Revised: 6 October 2021

Accepted: 7 October 2021

Available online: 22 November 2021
The article presents analysis of road crash accidents. It presents the evolution of safety systems, starting from a description of the currently used vehicle-based systems, with particular emphasis on the prediction of the driver falling asleep. The article also proposes a proprietary system of sleep prediction based on the face detection of drivers. The detection of facial landmarks is presented as a two-step process: an algorithm finds faces in general, and then needs to localize key facial structures within the face region of interest.

Key words: safety, system, vehicles, drowsiness, face detection

This is an open access article under the CC BY license (http://creativecommons.org/licenses/BY/4.0/)

\section{The analysis of road crash accidents}

\subsection{General information}

Each year more than 1.2 million people die in car related accidents around the world. Dying in a car accident is the most common cause of death among people aged between 15-29 years. According to statistics, roughly $90 \%$ of road traffic deaths occur in low- and middle-income countries. European roads remain the safest in the world, with a road traffic fatality rate of 9.3 per 100000 people. The world average is 17.4 per 100000 people, The worst results can be observed in the African Region, with a road traffic fatality rate per 100000 of 26.6 being almost three times higher than in Europe [1].

Such a difference is caused mostly by the fact that there are older cars, worse roads, a different lifestyle, ineffective police, and a poorer society. This is why the World Health Organization make 4 main recommendations in order to reduce road traffic fatalities:

1. Legal area: countries should create and enforce laws relating to risk factors.

2. Stricter enforcement of road safety rules: the need for special social campaigns to maximize effects.

3. Paying attention to pedestrians, cyclists, and motorcyclists: roads should be built in a way that every road user can feel and be safe.

4. Car safety: even though technology in the automotive industry has become advanced, cars in poor countries usually lack even the most basic safety features.

\subsection{Road accidents caused by drowsiness}

Tiredness can be described as a state of fatigue caused by, among others, physical activity, insufficient sleep, or sickness. Drowsiness is described as a tendency to fall asleep. There are three stages of the process involving sleep:

1. being awake

2. non-rapid eye movement (NREM) sleep, which can be divided into:

- stage 1: the transition from being awake to being asleep
- stage 2: light sleep

- stage 3: deep sleep

3. rapid eye movement (REM) sleep.

The stage of drowsiness that is most dangerous for road traffic users is stage 1 of the NREM stage. The sleepiness scale is also measured using the Karolinska sleepiness scale (KSS), the full version of which is presented in the Fig. 1. Drivers showing signs that are rated 6 or higher in the table are potentially dangerous.

\begin{tabular}{cc}
\hline Rating & Verbal descriptions \\
\hline 1 & Extremely alert \\
2 & Very alert \\
3 & Alert \\
4 & Fairly alert \\
5 & Neither alert nor sleepy \\
6 & Some signs of sleepiness \\
7 & Sleepy, but no effort to keep alert \\
8 & Sleepy, some effort to keep alert \\
9 & Very sleepy, great effort to keep alert, fighting sleep
\end{tabular}

Fig. 1. Karolinska sleepiness scale (KSS)

The National Highway Traffic Safety Administration, which is an organization operating in the United States, says that approximately 72000 road traffic accidents in 2013 were caused by the drowsiness of drivers. Those accidents resulted in 44000 injuries and 800 deaths. These numbers, however, could be underestimated, with the real number of fatalities being closer to 6000 [2].

Road accidents caused by tiredness can be more fatal. They usually occur at a higher speed, and as the driver is not vigilant, he has no possibility of braking or making other defensive maneuvers. The most common description of a road crash caused by drowsiness is an unexpected change of lane or the hitting of the car in front. Most fatigue-related accidents occur on motorways, which is due to the fact that there is almost no stimulus to the driver be- 
cause of the monotonous and boring environment. Moreover, many of the activities that are usually thought to help fight sleepiness (such as increasing the radio volume, opening windows, or pointing a blowing cold air supply at the driver's face) do not actually help at all.

According to research, falling asleep rarely comes without warning, but drivers tend to ignore the early signals, such as: difficulties with concentration, heavy eyelids, yawning, and dropping of the head. A very common phenomenon is microsleep, which is described as falling asleep for more than 2 , but less than 30 seconds without realizing it. The feeling the driver may have is that he thinks he was just nodding his head, even though he was really asleep.

As driver drowsiness is a common problem all over the world, there are many different ways to solve it. One quite popular and effective method is the painting of road lines in such a way that driving on them makes a very loud and unpleasant sound - so-called rumble strips. The aim of such strips is to wake up drivers if they begin to drift away from the correct lane.

To mitigate the results of highway accidents, special infrastructure is being built, e.g. crash barriers can absorb kinetic energy and keep the driver from hitting another element or an oncoming vehicle.

Car manufacturers are also trying to solve this problem, with driver fatigue detection systems becoming more and more popular.

\section{Existing safety systems}

\subsection{Active and passive safety}

Almost from the beginning of the automotive industry, car manufacturers have tried to improve their vehicles. The first recorded car accident happened in 1887, and from that date it can be said that road safety has also been important for producers.

Safety can be divided into that of active and passive. Passive safety is a set of vehicle features that attempt to minimize the effects of an accident. It can be divided into internal passive safety and external passive safety. Internal passive safety provides measures of reducing the probability of the driver or passengers being injured or killed, as well as the cargo being damaged. External passive safety concerns the safety of other road users, such as pedestrians. The most important passive safety devices are: seatbelts with proper regulation, child seats, airbags, and headrests. The following can also be seen to be important: the proper construction of the car's body, which absorbs kinetic energy in the event of a collision; proper placement of the gas tank; placing the car's battery outside of the crash zone; using safety glass; using proper door locks, which remain functional after the collision; using bendable steering shafts and a safe steering wheel: using energy-absorbing elements in the cabin; the reduction of pointy elements inside the car; and using non-flammable materials.

Active safety is a set of vehicle functions that either enable an accident to be avoided, or reduce the impact of the accident. It allows the driver to take preventive measures before an actual accident occurs. Many of the dynamic characteristics of a car can be related to active safety: the ability to reduce car slipping and drifting, the reduction of tire skids during braking, and the ability to accelerate on wet surfaces. The elements of active safety also include fog lights, good visibility, wipers, ergonomic seats, air conditioning, and an adjustable steering wheel. To improve a car's active safety, many subsystems and solutions are used in modern cars, for example:

1. Anti-lock Braking System (ABS), which prevents the wheel from blocking during intensive braking. Braking is performed in such a way that the grip of the wheels is as high as possible, with steering ability remaining intact.

2. Automatic Stability Requirement (ASR), which prevents the wheels from losing traction during acceleration. The system may use equipment provided by ABS, and also slow down the spinning of wheels due to a loss of traction.

3. Brake Assist, which is an emergency braking assistance system. If the system detects a sudden and strongly depressed brake pedal, it can automatically increase the brake fluid pressure.

4. Electronic Stability Control (ESC), which provides lateral stability. The system can take over the acceleration or braking required by the driver if it determines that the driver's actions are not correct. Corrections are usually made by braking one wheel in order to avoid actual skidding.

\subsection{Visual safety systems}

In 1956, during the General Motors Motorama, Buick company presented the first conceptual car with back-up cameras. Since then, the idea of a vision detection system has been developed by the automotive industry. When Toyota began offering a rear-view camera in its cars in 1997, the technology gained more and more popularity around the world.

Modern vehicles are usually equipped with not only one, but many cameras. An additional convenience for drivers is a system called Surround View. It can display a bird's eye view of the car. This is possible thanks to the many fisheye cameras mounted throughout the car.

Such cameras are used in the following safety systems:

1. Collision Avoidance System: its task is to prevent a collision or, if this is not possible, to reduce the severity of the collision. This system records images in front of the camera using radar, laser or a digital camera and, after processing the collected data, detects the possibility of an accident.

2. Pedestrian Detection System: enables the detection of pedestrians who may interfere with the car's path. The system can also be equipped with an infrared camera, which makes it easier to see pedestrians in dark surroundings.

3. Adaptive Cruise Control: a system that keeps the car at a constant distance from the car in front that is in the same lane and moving in the same direction.

4. Blind Spot Monitoring System: supports the driver by constantly monitoring the areas that are invisible to the driver. It can inform the driver if a car, which is not yet visible in the mirror, is overtaking him.

5. Lane Departure Warning System: warns the driver that the vehicle is about to leave the lane without the turn 
signals being activated. More advanced versions of this system can correct the trajectory to ensure the car stays in its lane, or even autonomously keep the car centered in the lane. The driver is only asked to take control if the lane markings are insufficiently visible.

\subsection{Driver tiredness detection systems in modern cars}

The driver drowsiness detection system is supposed to detect the first signs of driver fatigue and warn him about the necessity to take a break. If the systems are more advanced, they can autonomously stop the car in a safe manner when it detects that the driver is nodding or falling asleep.

Almost every major car manufacturer offers a system with a similar name and mission. Depending on the manufacturer, the main principle of operation is different. The basic division includes three groups: vehicle-based measured, behavior-based measured, and physiology-based measured [3].

Vehicle-based measurement systems analyze data from multiple sensors and try to detect any anomalies, such as: a change in pressure on the brake or accelerator pedal, steering wheel movements, or lane deviations. When the analyzed value reaches a certain level, the system will alert the driver. The following can be seen as examples of such systems:

1. Steering wheel motion monitoring: the system constantly checks the steering angle sensor and measures driver fatigue. If the driver becomes sleepy, the number of minor tweaks to the steering wheel is less than when the driver is alert. This solution usually only counts small corrections ranging from $0.5^{\circ}$ to $5^{\circ}$. Any wider correction can be considered a lane change rather than a course correction. The system is very sensitive when environmental issues appear. Even a minor defect in the steering system may interfere with the system measurements and give bad results. The poor condition of roads can also disrupt the proper operation of the system.

2. Standard Deviation of Lane Position: cameras mounted somewhere on the vehicle track the position of the car in the lane. If the driver is constantly changing lanes without using the turn signals, or drives between the lanes, the system assumes that the driver is sleepy and rest is advised. The system's greatest disadvantage is that it is prone to false results when environmental conditions are not good, like heavy rain, poor lane markings, snow, and poor lighting. The standard lane departure method can also be tricked into thinking that the driver is sleepy when driving under the influence of alcohol or drugs.

The driver drowsiness detection system based on behavioral measurements checks driver actions such as yawning, eye blinking, head position and the closing of eyes. Actions are recorded by a camera and then processed in order to determine if the driver should be alerted. When people get sleepy, they start to show many characteristic facial movements. These are frequent yawning, head tilting, head nodding, fast and constant blinking, and squinting. A noninvasive method of determining whether these symptoms are related to somnolence is to install a microcamera in front of the driver, to continuously monitor these behaviors, and to compare the collected data with normalized values.

The monitoring of driver tiredness may also be performed using physiological measures. There is some correlation between driver drowsiness and physiological symptoms such as electroencephalogram (EEG), electrooculogram (EoG), electromyogram (EMG), and electrocardiogram (EKG). The main benefit of this method is that it detects signs of tiredness before they affect the driver's ability to stay alert and drive safely. Nevertheless, the way this system works would require the driver to wear special equipment. It is seen to be more invasive than other solutions, so has never become popular and is not used in regular cars.

If we compare the top 20 car manufacturers, as in Table 1 , it can be seen that vehicle-based behavior systems are the most common. The popularity of this solution is due to the relatively low cost of implementing such a system. It allows the reuse of existing sensors and the data collected by other subsystems. Only 3 car manufacturers offer a Driver Drowsiness Detection System based on driver behavior: BMW Attention Assistant, DS Driver Attention Monitoring System, Jaguar and Land Rover Driver Status Monitor. The brands listed are generally considered to be luxurious in some way, and therefore their customers are willing to pay extra for more advanced and sophisticated systems.

Table 1. Comparison of the top 20 car manufacturers

\begin{tabular}{|c|c|c|c|}
\hline $\begin{array}{l}\text { Company } \\
\text { name }\end{array}$ & System name & $\begin{array}{l}\text { Vehicle } \\
\text { based }\end{array}$ & Behavior \\
\hline Audi & $\begin{array}{c}\text { Rest recommendation } \\
\text { system }\end{array}$ & $\mathrm{x}$ & \\
\hline BMW & Attentiveness Assistant & $\mathrm{x}$ & $\mathrm{x}$ \\
\hline Citroen & Driver Attention Alert & $\mathrm{x}$ & \\
\hline DS & $\begin{array}{l}\text { DS Driver Attention } \\
\text { Monitoring }\end{array}$ & & $\mathrm{x}$ \\
\hline Ford & Driver Alert & $\mathrm{x}$ & \\
\hline Honda & Driver Attention Monitor & $\mathrm{x}$ & \\
\hline Hyundai & $\begin{array}{c}\text { Driver Attention } \\
\text { Warning/Alert }\end{array}$ & $\mathrm{x}$ & \\
\hline $\begin{array}{l}\text { Jaguar/ Land } \\
\text { Rover }\end{array}$ & Driver Condition Monitor & & $\mathrm{x}$ \\
\hline $\mathrm{Kia}$ & Driver Attention Warning & $\mathrm{x}$ & \\
\hline Mazda & Driver Attention Alert & $\mathrm{x}$ & \\
\hline $\begin{array}{l}\text { Mercedes- } \\
\text { Benz }\end{array}$ & Attention Assist & $\mathrm{x}$ & \\
\hline GM Group & Driver Drowsiness Alert & $\mathrm{x}$ & \\
\hline Nissan & Driver Attention Alert & $\mathrm{x}$ & \\
\hline Renault & Fatigue Detection Warning & $\mathrm{x}$ & \\
\hline Skoda & $\begin{array}{c}\text { Fatigue Recognition } \\
\text { Assistant }\end{array}$ & $\mathrm{x}$ & \\
\hline Volkswagen & Driver Fatigue Detection & $\mathrm{x}$ & \\
\hline Volvo & Driver Alert Control & $\mathrm{x}$ & \\
\hline
\end{tabular}

\section{Proposal of a driver drowsiness detection system}

\subsection{General information}

The idea of the proposed system is to use real-time video of the driver, which is captured by a camera placed in front of him. Thanks to the image processing algorithm, it detects characteristic landmarks of the face, such as the time from last stop, eye opening level, blink time, head tilt, and excessive yawning. After detecting landmarks, the system calculates factors that may lead to the driver becoming drowsy. If the system determines that the driver is 
drowsy, the driver will be warned on the screen and played an alarming sound. It is important that the algorithm is properly optimized, because the analysis time cannot exceed the capture time of one video frame. Moreover, to ensure good image quality, the camera in the system captures video at 30 frames per second. The results provided by the system should be clear and precise for all drivers.

Such a system can be integrated with the car's safety and infotainment system, and therefore the results of driver drowsiness can be supplemented with information about keeping the car in one line, sudden movements of the steering wheel, and irregular use of the gas and brake pedals. Moreover, the system should persistently alert the driver that he is sleepy. If the driver does not rest after several alarms, the on-screen notification and alarm should become increasingly disturbing.

\subsection{Face detection systems}

Face detection systems first find the driver's face, and then locate more specific points. Descriptions of a few selected examples of such programs are shown below:

1. Face detection is the computer's ability to find a human face in a processed image thanks to certain patterns that can be seen on every face. A proper face detection algorithm should be able to find multiple faces in a photo, and not just find a face among the other elements of the photo. Parameter calibration is a key element of a properly functioning system.

2. Face recognition is the computer's ability to determine whether a processed face belongs to a specific person. Due to differences in faces, the algorithms can map the facial attributes during calibration, and then look for the distinctive attributes during recognition.

3. Face analysis is the computer's ability to read certain characteristics of a depicted face. Systems based on deep learning and machine learning can determine attributes such as age, gender, and even emotions.

4. Face tracking is the computer's ability to find specific facial characteristics in each frame of a processed video. Similar to face detection, algorithms learn to find patterns on the user's face. Distinctive points of the eyes, nose, mouth and chin can be specified.

This article is about a driver detection drowsiness system that focuses on face tracking.

\subsection{How the program finds a face in a picture}

Each digital image is made of pixels. A set of pixels of a given color (based on the RGB model) creates an image that can be stored in memory or displayed on the user's display. Therefore, the color of each pixel is described by a tuple of three numbers. In image processing, algorithms usually handle black and white images, which is due to the fact that color information is useless in most cases. Black and white images are described in grayscale, where (0) stands for black pixels, and (255) stands for white pixels, with all numbers in between being grayish.

The face detection algorithm was created in 2001 by Paul Viola and Michael Jones. The algorithm is divided into 4 main steps:

1. Selection of Haar-like features

2. Creating an integral image

\section{Running AdaBoost}

4. Creating cascades of classifiers

The algorithm scans the image and, looking at smaller areas, tries to find a face by looking for attributes in each area. Since the image can contain more than one face, and also have different sizes, the algorithm must be able to scale.

The Haar-like features make use of the properties seen in every human face captured in the photo. The most prominent feature is that the eyes are usually darker than the tip of the nose. To determine the brightness of the analyzed region, the algorithm adds the pixel values of each region. The lower sum tells the algorithm that the computed region is darker than the one with the larger sum. A visual representation of Harr-like features is shown by dividing a rectangular part of the picture, and then presenting it as black and white rectangles. Examples of it are shown in Fig. 2 below.

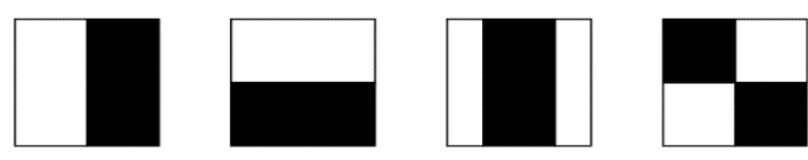

Fig. 2. Haar-like features used to detects patterns in images

Integral image (summed-area table) is an algorithm that speeds up the calculation of the sum of pixel values in a specific area. To compute the integral image, each pixel in a given area only needs to be processed once, and therefore only four numbers are needed.

With these calculated values, Adaptive Boosting (AdaBoosting) can be very useful. According to machine learning theory, a weak learner only classifies if a region contains a face slightly better than random guessing. Combining many weak learners together makes a strong learner, who is much better at finding faces. Adaptive Boosting is all about taking a plethora of weak classifiers and turning them into a strong classifier. The adaptive gain algorithm is applied to all image areas. Several areas of the image will give a higher score than others. These areas are classified as positive. For facial recognition, they are considered to include the face. Those considered not to contain a human face are classified as a minus. As the next iteration progresses, the negative elements gain more weight, and become more important for the classifier. The minus and plus areas change accordingly. After a few iterations, the classifiers are split equally, and the strong classifier is ready.

Image processing uses cascading classifiers. Cascade means that many subsequent behaviors are necessary to determine whether an image contains a face. The cascade is divided into many stages. If the first step of the cascade evaluates the region as positive, the region then moves on to further processing steps that are termed as "maybe". If either step evaluates a region as negative, the region is no longer processed and is considered to not contain a face.

\subsection{Eye recognition and blinking detection}

In order to detect objects on a detected face, the algorithm must use facial landmarks. The algorithm tries to find the required points on the face. Detecting facial landmarks is a two-step process. First, the algorithm finds faces in 
general, and then has to locate the key facial structures in the area of the face of interest. Most face landmark algorithms detect the following items: jaw, nose, left eye, right eye, left eyebrow, right eyebrow, mouth. The Dlib library used in this project to detect facial landmarks allows the location of 68 points of interest [4]. The points that are found are presented in Fig. 3.

If the classifier detects a facial landmark in an image containing a face, the result will be like in Fig. 4.

After detecting facial landmarks, it is possible to analyze whether the driver is sleepy. The clearest sign of sleepiness comes from the driver's eyes and blinking. Blink detection in this design is based on the so-called Eye Aspect Ratio (EAR). The previous algorithm is efficient enough to track 6 characteristic points for each eye. The proposed solution of using the EAR uses these 6 points for each and after simple calculations returns the aspect of the openness of the eye.

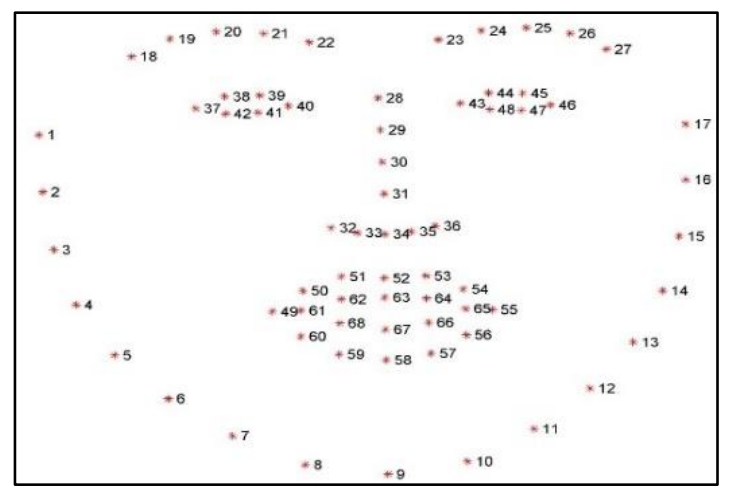

Fig. 3. Facial landmarks that are detected by the algorithm

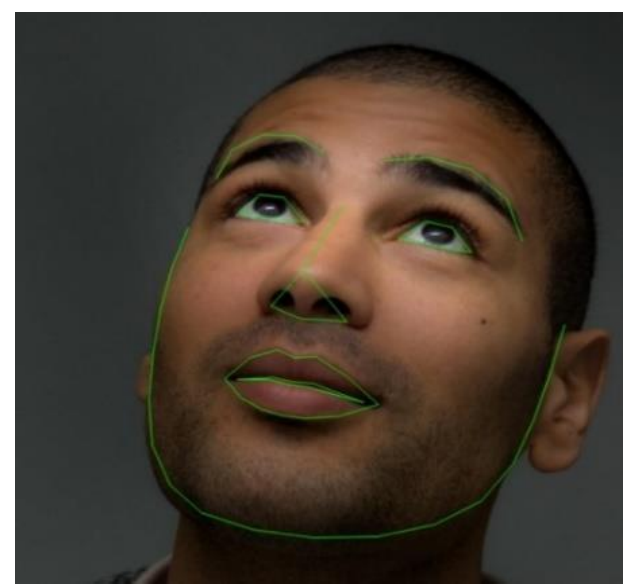

Fig. 4. Facial landmarks detected

According to research, there is a constant relationship between these points. Therefore, equation (1) can be derived in order to describe the proportions of the eye.

Equation (1) allows the distance between the vertical and horizontal landmarks of the eye to be calculated, which when properly weighted leads to the EAR. These landmarks are presented in Fig. 5.

$$
\operatorname{EAR}=\frac{\left\|p_{2}-p_{6}\right\|+\left\|p_{3}-p_{5}\right\|}{2 *\left\|p_{1}-p_{4}\right\|}
$$

When the eye is open, the proportions of the eye are almost constant. However, when the eye is closed, the number almost drops to zero. It is safe to assume that the EAR dropping below a certain threshold corresponds to the user blinking. Using the eye aspect ratio is also useful for detecting driver drowsiness. Because the proportions of the eye drop below a certain level and do not change their value for a long time, the blinks exceed the usual 100-400 ms. Over time, it can be assumed that the driver becomes drowsy.

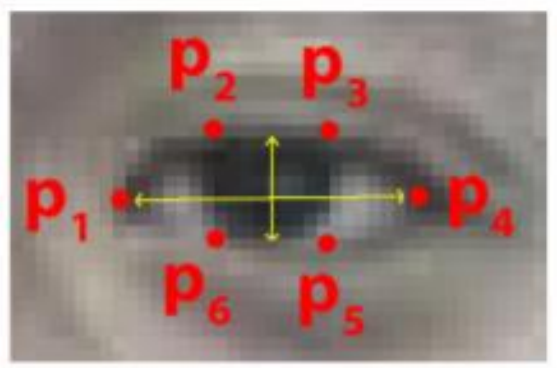

Fig. 5. Landmarks for an opened and closed eye

The algorithm used in the created driver tiredness detection systems can be divided into two stages:

1. Calibration phase: it is assumed that the driver starts the journey fully awake, and therefore the mean values of the parameters are calculated and used later as a model.

2. The system calculates the driver's drowsiness by comparing the actual values with the reference values collected during the calibration phase.

The average value of the EAR is calculated during the calibration, and if its value drops by more than $40 \%$ it is assumed that the driver is sleepy. The system calculates the blinking frequency every 10 seconds. The algorithm also records the time of a single blink. It is one of the most important parameters in determining drowsiness. Tilting of the head is also considered to be one of the factors of driver tiredness. An asymmetrical head position adds points to your overall drowsiness score.

\subsection{Example of how the system works}

Calibration takes place each time the system is started. This is important, as lighting conditions can vary and a different driver may be seated in the seat. In the calibration mode, the system displays research and development data, such as the number of blinks, blink duration, eye aspect ratio, face length, face position and blink frequency. The default calibration time is 60 seconds, which is enough for development purposes. In real-world situations it should be increased to at least 5 minutes for better data collecting. After calibration, all results are averaged and stored in the system's memory for reference. It is possible to recalibrate the system in the event of a malfunction. During calibration, the user's eye and nose are highlighted, with the mean value of the EAR obtained during calibration being between 0.30 and 0.35 .

After calibration, the system will start detecting driver drowsiness. The parameters obtained from the captured video frames are compared with the reference values. Each parameter is properly weighted so as not to distort the final result. The most important parameters are the time of 
a single blink and the proportions of the eye. These parameters can detect if a driver is sleepy or not. Closing eyes for more than one second greatly increases the effect of sleepiness. A special smile detection algorithm can lower the eye aspect threshold, as people tend to squint when smiling. Parameters such as yawning and tilting the head are weighted as less important. Paying too much attention to tilting your head can cause many false positives. Safe driving requires the driver to frequently check the mirrors, and this may cause some drivers to tilt their head slightly.

When the system remains active, with developer mode enabled, it displays all calculated information on the image. This means that it is possible to see if all the calculated information is correct. The most important parameter for the system remains the calculated fatigue value. This is the final result of the system, and ranges from 0 to 300 . When the value is close to 0 , the driver is considered fully alert. When the value exceeds 120 , the system will consider the driver to be drowsy. Information appears on the screen and an audible alarm sounds.

An example of when the driver is alert is visible in Fig. 6, with Fig. 7 showing the driver when he is tired.

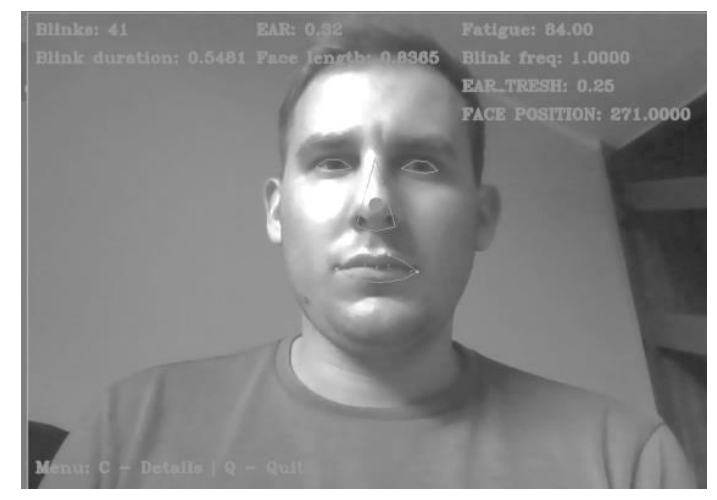

Fig. 6. User's interface when the driver is alert

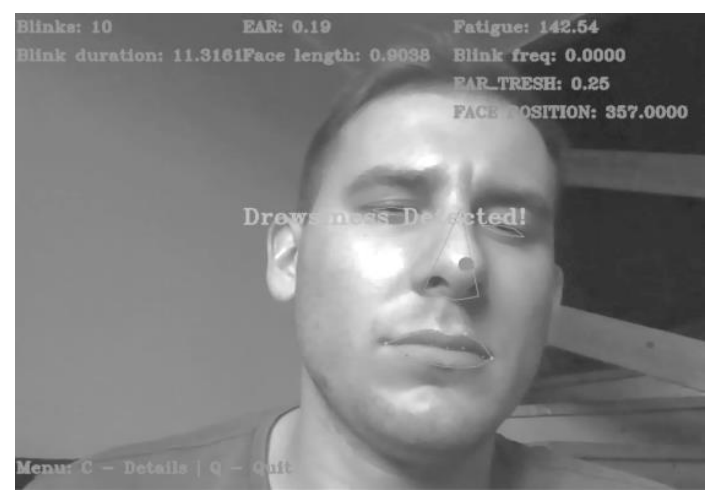

Fig. 7. User's interface when the driver is drowsy
In Figure 6, the fatigue level is 84 (visible in the top right corner). The proportion of the eye is high, and this is one of the main elements that has an influence on the final result. In Figure 7, the fatigue level is 142.54 (visible in the top right corner), and therefore the alarm is shown on the screen. When the fatigue detection is over, the system returns a graph of the recorded fatigue level for the entire period of activity. This allows the values recorded by the system at peak times to be better analyzed. Any anomalies, such as sudden changes in lighting or looking for upcoming traffic, which may cause different result values, are visible in the charts. When the system loses track of the driver's line of sight, the fatigue value remains constant until sight is found and tracked again.

An example of the mentioned chart can be seen in Fig. 8. The analysis of this graph may bring some conclusions about the operation of the system and its effectiveness. The first seconds of work are needed to determine the appropriate level of driver drowsiness. After about 50 seconds, the system gets a fairly consistent result, which is set to around 90. This level of tiredness is considered by the system as "being alert". Up to a peak of around 270 seconds after takeoff, there are some dips in fatigue. It gets values of around 65. This may be due to a change in lighting or wider eyes, or could actually be a real result. After the peak has caused the driver to look around, or squinttheir eyes to see traffic lights or avoid direct sunlight, the driver's fatigue level returns to its previous level and becomes stable (ranging from around 65 to 80 ).

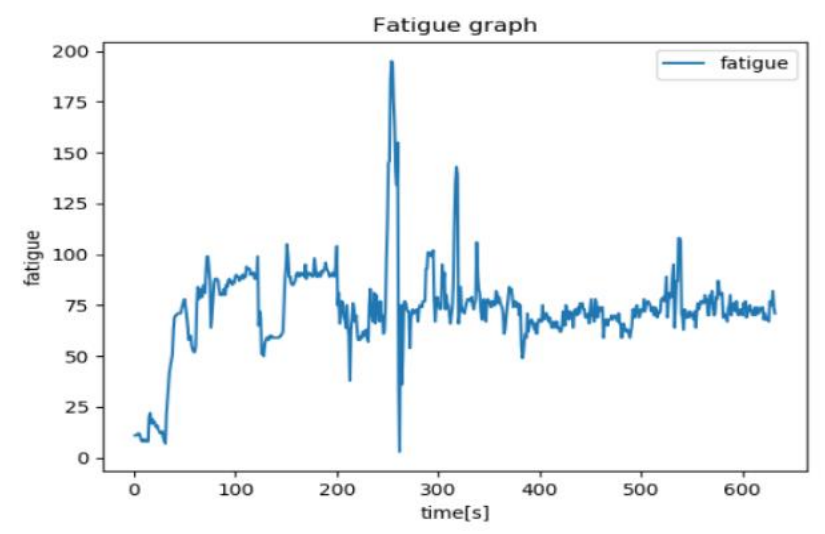

Fig. 8. Chart of the recorded fatigue level. The driver was alert

Despite the already existing systems of this type on the market, there are still many possibilities for their improvement or modification.

The experimental system mentioned in the article was built and tested at the Department of Vehicle Engineering at the Wrocław University of Technology.

\section{Nomenclature}

ABS Anti-lock Braking System

ASR Automatic Stability Requirement

EAR Eye Aspect Ratio

ESC Electronic Stability Control
KKS Karolinska sleepiness scale

NREM non-rapid eye movement sleep

REM rapid eye movement sleep 


\section{Bibliography}

[1] Global Status Report on Road Safety. World Health Organization, 2018.

http://apps.who.int/iris/bitstream/handle/10665/277370/WH O-NMH-NVI-18.20-eng.pdf?ua $=1$

[2] Centers for Disease Control and Prevention. Information about drowsy driving risks.

https://www.cdc.gov/features/dsdrowsydriving/index.html

[3] SAŁAPATEK, D., DYBAŁA, J., CZAPSKI, P. et al. Driver drowsiness detection systems. Proceedings of the Institute of Vehicles. Warsaw University of Technology. 2017, 3(112), 41-48.

[4] PyImageSearch. Information about face elements detection. https://www.pyimagesearch.com/2017/04/10/detect-eyesnose-lips-jaw-dlib-opencv-python

Piotr Haller, MEng. - Faculty of Mechanical Engineering, Wroclaw University of Technology.

e-mail: piotr.haller@pwr.edu.pl

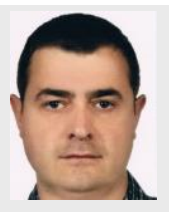

Prof. Radoslaw Wrobel, DSc., DEng. - Faculty of Mechanical Engineering, Wroclaw University of Technology.

e-mail: radoslaw.wrobel@pwr.edu.pl

Gustaw Sierzputowski, DEng. - Faculty of Mechanical Engineering, Wroclaw University of Technology. e-mail: gustaw.sierzputowski@pwr.edu.pl

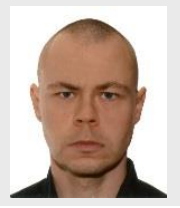

[5] LEMOV, M.R. Car Safety Wars: One Hundred Years of Technology, Politics, and Death. Fairleigh Dickinson University Press. 2015.

[6] ROSS, H.L. Functional safety for road vehicles. Springer 2015. https://doi.org/10.1007/978-3-319-33361-8

[7] OKA, D.K. Building Secure Cars: Assuring the Automotive Software Development Lifecycle. Wiley. 2021. https://doi.org/10.1002/9781119710783.ch3

[8] LOTHAR, W., SEIFFERT, U. Automotive Safety Handbook. SAE International. 2007. https://doi.org/10.4271/R-377

[9] AGARWAL, A., YADAV, V. Advanced integrated future vehicle telematics system concept modelling. Global Journal of Technology \& Optimization. 2017, 8(2), 1-6. https://doi.org/10.4172/2229-8711.1000215

Radostin Dimitrov, DEng. - Faculty of Mechanical Engineering, Varna University of Technology.

e-mail: r_dimitrov@tu-varna.bg

Veselin Mihaylov, DEng. - Faculty of Mechanical Engineering, Varna University of Technology.

e-mail:v_mihaylov@tu-varna.bg 